\title{
The Hypothesis of Impact Origin of Diamonds and Kimberlites
}

\author{
Alexey M. Lyukhin \\ Russian state geological prospecting university, Moscow, Russian Federation
}

Despite a large volume of accumulated actual material the problem of origin of kimberlites and diamonds still remains unclear. It is reflected in the existence of a number of hypotheses differently and frequently alternatively ascribing the principal aspects of processes of natural kimberlite and diamond formation. Neither has been finally resolved the mechanism of formation of pipes themselves. The substantiation of a cause-and-consequence link, the conditions and mechanisms of formation of diamonds and all types of diamondiferous rocks is possible in the framework of an impact process - one of the most fundamental geological processes brought about in the Solar system.

The impact hypothesis assume that a great space catastrophe - the tenth planet of the Solar system Faeton collided with a gigantic interstellar comet about $3,2-3,4$ billion years. It is during this collision and the follow-up explosion that the formation of a major mass of diamonds currently found in meteorites and kimberlites is likely to have taken place. The major key moments of diamond genesis are as follows: the source of carbon, pulse P-T conditions to form diamond seeds, continuous feeding by carbonaceous matter at high temperature and pressure to ensure the growth for a certain time and an availability, upon the completion of growth, of certain conditions to preserve the crystals once formed. Except for the high temperature/pressure, the mantle hypothesis is not able to give an exactly answer to these questions. But in view of the suggested hypothesis all conditions are met. Taking into account the composition of comets $-\mathrm{H}_{2} \mathrm{O}, \mathrm{CO}_{2}, \mathrm{CH}_{4}, \mathrm{NH}_{3}, \mathrm{C}_{3}$, $\mathrm{C}_{2}, \mathrm{CN}, \mathrm{CH}, \mathrm{OH}, \mathrm{NH}, \mathrm{NH}_{2}$ the source of carbon for the formation of coaly chondrites, ureilites and diamonds, the source of nitrogen in their crystalline lattice and the composition of gaseous inclusions are logically substantiated. The duration of a process of impact collision of even big meteoroids with the Earth makes from parts of seconds to several seconds. During this period only small crystals up to $1 \mathrm{~mm}$ in size can be formed. But the collision of a planet with a comet is quite another thing. The mass and density of a comets' core are inconsiderable, but the length of their tail reaches many millions of kilometers. When a planet and a comet collide, even keeping in mind their great speeds, the process of the shock collision, explosion and disintegration of the planet may last several hours, and during this time, in view of its scope, crystals much larger than that of Cullinan (3106 carats) could be grown. One can easily explain a great variety of morphological and crystallographic forms, sizes, color of diamond crystals as well as the composition and morphology of solid inclusions, the isotopic composition of helium and many other things resulting from such a process. The composition of gaseous inclusions in diamonds is not characteristic of the Earth's mantle what is acknowledged by the authors themselves of the mantle hypothesis of kimberlites origin. J. Dawson writes: "Nitrogen is concentrated in the form of thinnest plates and in single centers inside a diamond, and it happens to be the only diamond location in the upper mantle known today. Should any though scarce data exist in respect of the sources of carbon and phosphorus, then the primary source of nitrogen and other rare gasses (e.g. ethylene, butane) in diamond remains a puzzle".

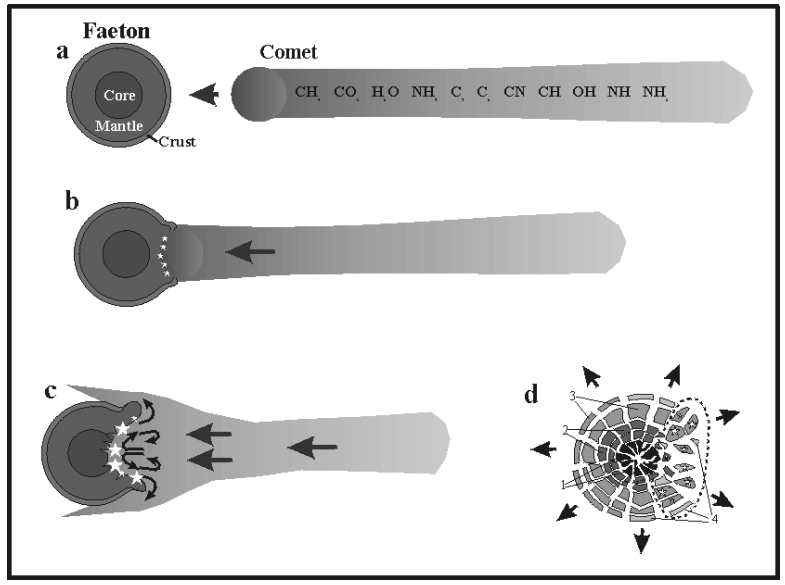

The schematic illustration of Faeton's collision with a galactic comet. (a - the approach of a comet toward Faeton; $b$ - the collision of space bodies, the generation and the beginning of growth of diamond crystals (1-st stage of diamond crystals growth after Frank); $\mathrm{c}-$ the turbulent growth of diamond crystals at the expense of the comet's tail's substance moving with the initial speed (2-nd stage of growth after Frank); d - the explosion of Faeton and the formation of iron, ironstone, stone and coaly meteoroids (the dotted line indicates the area of distribution of meteoroids with diamonds).

As a result of collision Faeton exploded and disintegrated into fragments some of which (akin to ureilites) carried diamonds. It is these fragments that 
are a "conserving" and "transporting" medium for diamonds. The absolute age of most diamonds is more than 3 billion years, and it is recognized by the majority of scholars in the whole world. It is hard to imagine the conditions to preserve diamond crystals in the mantle for billions of years but in the outer space they could have been preserved in the initial state for as long as possible. One part of the fragments - formed a belt of asteroids between Mars and Jupiter. The other part ran into the field of gravitation of other planets and became their satellites. The third part - subjected the Solar system's large cosmic bodies to an intensive meteorite bombardment in the nearest time after the catastrophe. Finally, the fourth portion of the fragments keeps on moving within the Solar system at their orbits up to this time periodically making collisions with the Earth and other planets of the Solar system.

The process of kimberlite formation takes place as a result of an impact interaction of stone meteoroids with the atmosphere and rocks of the upper part of the Earth's crust. Passing through the Earth's atmosphere most meteoroids get disintegrated. The disintegration of meteoroids does not actually depend on their sizes: the aerodynamic forces are the same for their both large and small representatives. As the major body of a meteoroid disintegrates its side-trended expansion takes place in which connection the overall area of cross section and, consequently, the resistance increases what causes a further growth of stresses. After the meteoroid has passed through the atmosphere the most important and necessary condition to form kimberlite bodies is the shape of its frontal part at the moment of touching the Earth's surface. During the aerodynamic disintegration of the meteoroid its sidecoursed expansion is brought about as a result of opening the fractures in the frontal part and removing exfoliated material sideways. At this time the stresses are maximum exactly in the places of fractures intersection what leads to the formation of cone-shaped cavities (cumulative hollows) that cause a cumulative effect at the moment of collision leading to the formation of hypersonic jets of strongly compressed meteoroid's substance. This is yet the only physical process in nature capable to create cavities of a pipe type in a solid medium.

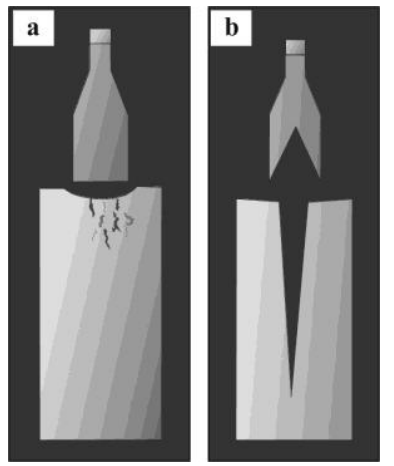

Kinds of destruction brought about in a steel target by action of: $a$ - full charge; $b$ - charge with a cumulative hollow.
The cone- or pyramid-shaped isometric hollows create pipes isometric in plan, the cone-like hollows of irregular shape create the same forms of pipes quaint in plan, the adjoined hollows form paired pipes, the linear and arch fractures opened at an acute angle - create linear and ring dykes and the like. Figuratively speaking, each pipe has its individual "face". Contrary to a cumulative charge, the rear meteoroid's part continuing to move on after the touch with the initial speed, since it is not yet subject to the processes taking place in the frontal part, plays the role of detonator here (and the source of a detonation wave); and the stone material of the hollow's walls is a shell. The formed high-speed jet (jets) breaks through terrestrial rocks forming a pipe-like cavity. Subject to the shape and sizes of the hollow the cavity's depth could make from tens of meters to first kilometers. Reaching a certain depth, as the energy recedes, the jet's substance either fills a fracture in host rocks or pinches out. The cooled jet's material having filled the lower parts of pipes and dykes represents a intrusive kimberlite with melted monocrystals of refractory minerals. This explains an intensive thermal contact of bottom parts of pipes with host rocks.

A general process of impact crater-formation takes place further on the surface. At the stage of contact and compression, as a result of conversion of the meteoroid's kinetic energy into internal energy the substance of the meteoroid and terrestrial rocks is exposed to significant shift deformation, full or partial fusion. Subject to the sizes, mineral composition, structure and jointing of various meteoroid's parts the partial fusion comes about unevenly (the inside nonmelted fragments further form xenoliths of "related" inclusions). By effect the impact of a high-speed meteoroid looks like a nuclear explosion, and in this very moment does the expansion of the upper part of a pipe-shaped cavity with the formation of a funnel come about. At the stage of excavation a hot cloud consisting of vapor, small particles, melted refractory crystals and fragments of rocks (xenoliths) slowly rises acquiring a classical mushroom-shaped form. It is this cloud where such specific formations of kimberlites as silicate globules and autoliths take place. The filling of the cavity formed by a high-speed jet begins at the stage of modification. A mixture of shock-formed melt with fragments of crater-hosting rocks and related inclusions, sometimes with large blocks ("floating reefs") drains down into it from the bottom of the crater; the small particles, fragments and autoliths lifted by vapor fall down from top - a kimberlite breccia gets formed. Separate fluxes of such melt form the so-called kimberlite "columns" or "intrusion phases" observed in many pipes. The hybrid composition of kimberlites, the absence of distinct contact alterations of host rocks and xenoliths in the diatremic parts of pipes, the gliding planes on a contact with host rocks, the variety of forms of pipes in plan, the availability and composition of crater facies in the upper parts of pipes and many other things are also easily explained. Wide variations of the chemical 
composition of kimberlite bodies are explained by a variety of compositions of meteoroids and terrestrial rocks. The distribution of diamonds in the fragments of an outer space body was extremely irregular from what one may easily explain an availability of diamondiferous and non-diamondiferous kimberlite bodies. And the epochs of kimberlite origination are associated with the Earth's passing through swarms and flows of asteroids at certain periods of space time.

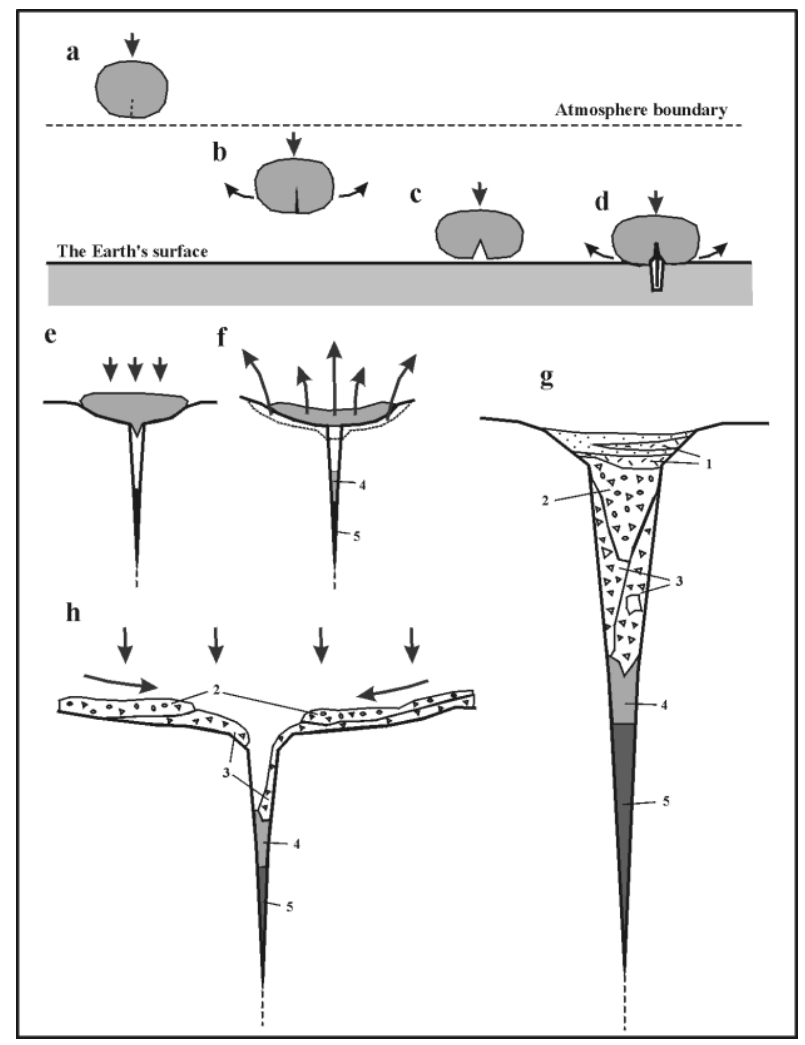

The schematic model of the impact formation of kimberlitic pipes. a - the form of a meteoroid before entering the atmosphere; $b$ - aerodynamic expansion of the meteoroid, the opening of a joint in its frontal part; $\mathrm{c}$ - the form of the meteoroid before the impact, the joint in the frontal part got disclosed and attained the form of a cumulative hollow; $d$ - the first moment of collision, the beginning of the formation of a cumulative spout; e - the spout punched the upper part of the Earth's surface, the spout's material had filled the lower part of the formed hollow; $\mathrm{f}$ - an explosion and the beginning of an excavation stage, the formation of a funnel of the pipe (part of the meteoroid's substance before the explosion might manage to get into the formed cavity); $\mathrm{g}$ - the beginning of a stage of shock crater modification, the flows of a mixture of breccias and melt fill the pipe, from top falls the material lifted by a sultan of outbursts, the lapillic (or autolitic) breccias will be the last facies to fill the pipe; $\mathrm{h}$ - the final shape of the explosion pipe.

Rocks of the pipe: 1 - tuffogenic-sedimentary formations of a crater lake; 2 - autolitic kimberlitic breccias; 3 - breccias and tuffo-breccias of several varieties of kimberlites; 4 - "intrusive" kimberlitic breccias; 5 - "intrusive” massive kimberlites.
The availability of dense atmosphere essentially changes the process of impact crater origination. The craters made by a destroyed intruder are small in comparison with those created by an undestroyed asteroid and are characterized by almost a flat floor. The "bushes" of kimberlite bodies and the kimberlite fields are likely to get originated from an impact interaction of such large (more than $1 \mathrm{~km}$ ) stone meteoroids subjected to aerodynamic destruction with the Earth's surface. Figuratively speaking, a kimberlite field is a preserved negative print of the surface of a meteoroid having collided with the Earth. The absence of any structural control over their location as well as an isometric and ellipse-like form in plan of the contours of kimberlite fields testify in favor of it. During tens and hundreds of millions of years the craters were destroyed by erosion, only the ring structures comparable with the craters' dimensions testify to their existence.

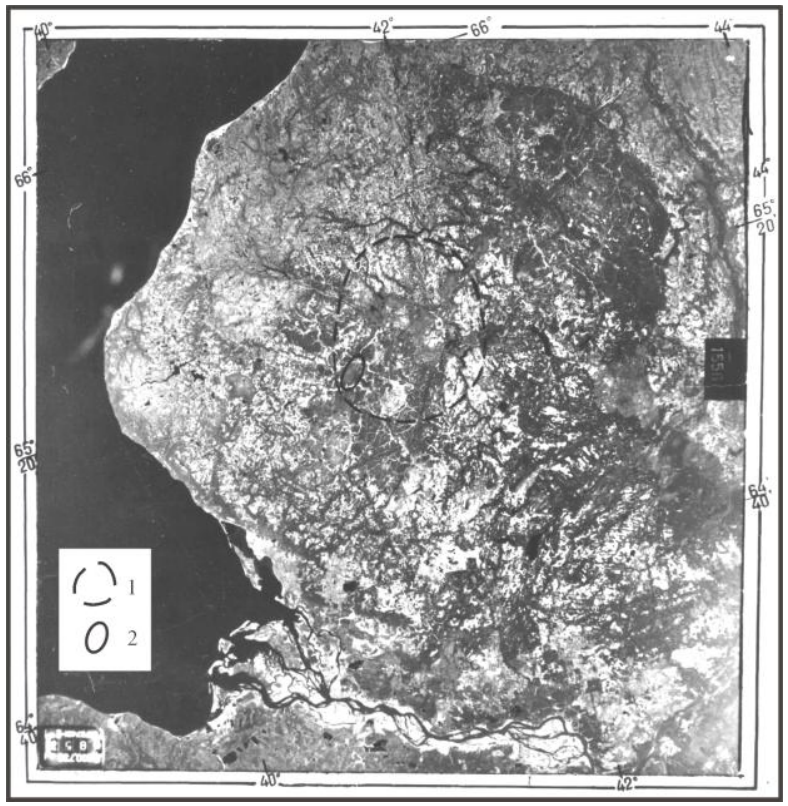

Ring structures round kimberlite fields in the area of Zimniy bereg (Russia, the Arkhangelsk Region).

(1 - a Zimniy-bereg kimberlite area, 2 - a Zolotitsk kimberlite field).

In conclusion, the term itself - "pipes of explosion" should be mentioned. In view of the origin from the Earth's depths, it did not correspond much to the facts and was often subjected to criticism. In view of the impact origin, one has to be surprised how much prophetic this name was since the impacts of supersonic meteoroids are really like explosions.

\section{References}

Dawson, J. B., 1980. Kimberlites and Their Xenoliths. Springer-Verlag Berlin-Heidelberg-New York.

Melosh, H.J., 1989. Impact Crtaering: A Geological Process. Oxford University Press, New York. 